\title{
Erythropoietin Improves Functional and Histological Outcome in Neonatal Stroke
}

\author{
YUN SIL CHANG, DEZHI MU, MICHAEL WENDLAND, R. ANN SHELDON, \\ ZINAIDA S. VEXLER, PATRICK S. MCQUILLEN, AND DONNA M. FERRIERO
}

\begin{abstract}
Department of Neurology [Y.S.C., D.M., R.A.S., Z.S.V., D.M.F.], Department of Radiology [M.W.], Department of Pediatrics [P.S.M.], University of California San Francisco, San Francisco, California, 94143, Department of Pediatrics [Y.S.C.], Samsung Medical Center, Sungkyunkwan University School of
\end{abstract} Medicine, Seoul, Korea

\begin{abstract}
ABS
Neonatal stroke is a condition that leads to disability in later
life, and as yet there is no effective treatment. Recently, eryth-
ropoietin (EPO) has been shown to be cytoprotective following
brain injury and may promote neurogenesis. However, the effect
of EPO on functional outcome and on morphologic changes in
neonatal subventricular zone (SVZ) following experimental neo-
natal stroke has not been described. We used a transient focal
model of neonatal stroke in P10 rat. Injury was documented by
diffusion weighted MRI during occlusion. Immediately upon
reperfusion, either EPO (5U/gm) or vehicle was administered
intraperitoneally and animals were allowed to grow for 2 wk.
Sensorimotor function was assessed using the cylinder rearing
test and then brains were processed for volumetric analysis of the
SVZ. Stroke induced SVZ expansion proportional to hemispheric
\end{abstract}
Stroke is recognized in about 1 in 4000 full-term infants (1). Stroke during the perinatal or early postnatal period affects CNS development and leads to lifelong neurologic morbidity such as cerebral palsy. However, there are as yet no clear evidence-based strategies for treatment (2).

We previously developed a nonhemorrhagic ischemic stroke model in immature rat by transient $\operatorname{MCAO}(3,4)$, which can be used for testing treatment strategies for neonatal ischemic stroke. Recent studies indicate that EPO plays an important role in neuronal survival after injury in a variety of models in mature and immature rodent brain. Not only direct intracerebroventricular injection (5-7) but also systemic administration of rhEPO significantly reduced the infarct volume following MCAO in adult rodent $(8,9)$ and in a hypoxia-ischemia model in the neonatal animal (7,10-12). Permanent focal brain isch-

Received August 23, 2004; accepted November 15, 2004.

Correspondence: Donna M. Ferriero, M.D., Departments of Neurology and Pediatrics, University of California San Francisco, 521 Parnassus Ave., C215, San Francisco, CA 94143-0663; e-mail: dmf@itsa.ucsf.edu

Supported by a grant (NS 35902) from Johnson \& Johnson Pharmaceutical Research and Development (Raritan, NJ), and by Sungkyunkwan University School of Medicine and Samsung Medical Center, Seoul, Korea 50 I/won-dong, Kangnon-ku 135-710.

DOI: 10.1203/01.PDR.0000163616.89767.69 volume loss. EPO treatment markedly preserved hemispheric volume and decreased the expansion of SVZ unilaterally. Furthermore, EPO treatment significantly improved the asymmetry of forelimb use following neonatal stroke. This functional improvement directly correlated with the amount of preserved hemispheric volume. These results suggest EPO may be a candidate in the treatment of neonatal stroke. (Pediatr Res 58: 106-111, 2005)

Abbreviations
EPO, erythropoietin
MCAO, middle cerebral artery occlusion
rhEPO, human recombinant erythropoietin
SVZ, subventricular zone

emia activates EPO receptor in neonatal brain (13) and exogenous rhEPO is protective in that model (14), suggesting EPO may play an important role in ischemic injury in developing brain.

To be a promising neuroprotective therapy, not only improved histology but also improved neurologic function has to be demonstrated. Kumral et al. (15) reported that systemic administration of EPO in neonatal animals immediately after cerebral hypoxia-ischemia improved long-term spatial memory deficits. However, there is as yet no published data for the effect of EPO on brain protection and on improving sensorimotor deficit after pure ischemic stroke in the neonatal animal.

EPO is known to promote neurogenesis in vitro (16), and recently Wang et al. (17) reported EPO enhanced neurogenesis in SVZ after stroke in the adult rodent. The SVZ in rodent forebrain is the germinative area for newly generated olfactory bulb neurons throughout life (18). Focal ischemic injury stimulates precursor proliferation and increases the size of adult rodent forebrain SVZ (19,20). Regarding the SVZ of neonatal rodent brain, little is known and controversies exist. Some studies have reported depletion of neural stem cells in SVZ after hypoxia-ischemia $(21,22)$. On the contrary, a recent report 
from Plane et al. (23) showed that hypoxia-ischemia in neonatal mouse augmented the SVZ size similar to the adult. Furthermore, they showed that severity of injury induced by hypoxia-ischemia is directly related to the degree of expansion of neonatal SVZ.

The changes in neonatal SVZ after pure ischemic stoke in newborn animals are unknown, and the effect of exogenous administration of EPO on neonatal SVZ has not been demonstrated. Therefore, we performed analysis of neonatal SVZ volume and sensorimotor function after focal ischemiareperfusion injury in immature postnatal day $(\mathrm{P}) 10$ rats treated with EPO or vehicle to elucidate the response of the neonatal SVZ to ischemic injury and to investigate the therapeutic potential of exogenous EPO in neonatal ischemic stroke.

\section{METHODS}

All animal research was approved by the University of California, San Francisco Institutional Animal Care and Use Committee and was performed in strict accordance with the relevant National Institutes of Health guidelines. Every effort was made to minimize animal suffering and to reduce the number of animals used.

Cerebral focal ischemia: reperfusion and treatment. All surgical procedures were performed in 10-d-old Sprague-Dawley rats. P10 rats were chosen for this neonatal stroke model to more accurately approximate the development of the term human newborn (24). Female Sprague-Dawley rats with an 8-9-d-old litter (9-10 pups per litter) were bought from Simonson Labs (Gilroy, CA). The mother was given food and water and housed in a temperature- and light-controlled facility until the pups were $10 \mathrm{~d}$ old. Transient focal cerebral ischemia was produced using the MCAO model with some modifications, as published before $(4,25)$. Briefly, each pup was weighed and anesthetized with $3 \%$ isoflurane in a mixture of $70 \% \mathrm{~N}_{2} \mathrm{O}$ and $30 \% \mathrm{O}_{2}$. After induction of anesthesia, $1.5 \%$ isoflurane was maintained. Because P10 pups have not developed thermoautoregulation, rectal temperature was monitored and maintained at $36-37^{\circ} \mathrm{C}$ with a combination of overhead lights and a heating blanket. With the animal supine, the right common carotid artery (CCA), external carotid artery (ECA), and internal carotid artery (ICA) were exposed with a midline cervical incision. The pterygopalatine, occipital, superior thyroid, maxillary, and lingual arteries were coagulated. After ligation of the ECA, a 5-0 nylon monofilament suture with blunted tip was inserted into the ECA lumen and gently advanced through the ICA up to the middle cerebral artery until slight resistance was felt. After placement of the suture, the wound was closed. Sham controls did not have the suture advanced.

Magnetic resonance imaging. Each animal was examined by diffusionweighted spin echo planar imaging (SE EPI) at 20-30 min after MCAO to eliminate animals with inadequate or atypical injury patterns. The entire brain was imaged with serial $20 \mathrm{~mm}$-thick coronal sections as previously described (3) using the following pulse sequence settings: TR/TE $=5000 / 60 \mathrm{~ms}, 4$ averages, field of view $(\mathrm{FOV})=35 \mathrm{~mm}$, data matrix $128 \times 128$, diffusion gradient duration $=20 \mathrm{~ms}$, separation $=29.7 \mathrm{~ms}$, amplitude $=70 \mathrm{mT} / \mathrm{m}$, b-factor $=1045 \mathrm{~s} / \mathrm{mm}^{2}$. Pups that exhibited ischemic injury in atypical regions, such as brainstem, or that showed lack of cortical involvement were excluded from the study. In a preliminary study, we found that an occlusion time of 45 min produced a moderate level of injury. Reperfusion was achieved after $45 \mathrm{~min}$ of occlusion by removing the suture under general anesthesia. Two of the MCAO animals were imaged during the transit of bolus-injected DyDTPA-BMA before and $20 \mathrm{~min}$ after removing the suture to confirm complete disruption of cerebral blood flow during occlusion and partial restoration of blood flow after reperfusion.

Immediately upon reperfusion, either vehicle $[0.1 \%$ BSA (Sigma Chemical Co., St. Louis MO) in saline] or rhEPO (a generous gift from Johnson \& Johnson) at a dose of 5 units per gram of body weight were injected intraperitoneally. Sham animals were also injected with vehicle or EPO after sham surgery. After the injection, animals recovered from anesthesia and were returned to their dam for $2 \mathrm{wk}$.

Sensorimotor function: cylinder test. All rats were evaluated for sensorimotor asymmetry at $2 \mathrm{wk}$ after surgery (P24) with the cylinder test (26). Rats could explore the walls of the cylinder as early as P21 (26). The apparatus was a cylinder of transparent Plexiglas and measured $20 \mathrm{~cm}$ in diameter and $30 \mathrm{~cm}$ in height (27). The cylinder was high enough so that the animal could not reach the top edge of the apparatus by rearing. The width of the cylinder allowed the animal to be freely moving horizontally. The cylinder was sufficiently heavy that it did not move when the animal supported its weight against the wall. Animals were handled for about $10 \mathrm{~min}$ per day $3 \mathrm{~d}$ before testing. Each animal was individually placed in the cylinder and observed for $3 \mathrm{~min}$. Initial forepaw of each weight-bearing contact with the wall during a full rear was recorded, or "both" were recorded for simultaneous contact with both forepaws. Nonimpaired (right, ipsilateral) or impaired (left, contralateral) percentage of total forepaw contacts at initiation was calculated. Percentage difference between nonimpaired and impaired paw initiation was calculated as the percentage nonimpaired initiation subtracted from the percentage impaired forepaw initiation. All scoring was done by raters who were unaware of treatment group assignment. All animals were scored twice by two independent raters and the average scores of the two raters were used for data analysis.

Histology. For histopathologic examination, after cylinder testing, animals were anesthetized with sodium pentobarbital $(100 \mathrm{mg} / \mathrm{kg})$ (Nembutal, Abbott Labs, Abbott Park, IL), and killed at P24 by transcardiac perfusion with ice-cold 4\% paraformaldehyde in $0.1 \mathrm{M}$ PBS ( $\mathrm{pH}$ 7.4). Brains were carefully removed and postfixed overnight, equilibrated in $30 \%$ sucrose in $0.1 \mathrm{M}$ PBS and left at $4{ }^{\circ} \mathrm{C}$ up to a maximum of $72 \mathrm{~h}$. Free-floating serial 40 um coronal sections were collected throughout the brain in each animal on a freezing microtome. The sections were stored at $4{ }^{\circ} \mathrm{C}$ in $0.1 \mathrm{M}$ phosphate buffer with $0.1 \%$ sodium azide for a maximum of $2 \mathrm{wk}$ and then stored in cryoprotectant at $-20^{\circ} \mathrm{C}$ until staining. The mounted sections were air-dried, stained with cresyl violet, dehydrated in graded ethanol solutions, cleared in Citrosolv (Fisher Scientific, Pittsburgh, PA), and cover-slipped in Permount (Fisher Scientific).

Stereologic volumetric analyses of bilateral hemisphere and SVZ. Using systematic random sampling, a series representing every 12th section was selected, cresyl violet stained, and analyzed. All volumetric quantifications were performed with a Nikon Eclipse E600 photomicroscope equipped with a high-resolution CCD camera, a motorized XYZ axis computer-controlled stage, and Neurolucida and Neuroexplorer morphometry software package (MicroBrightField, Inc., Colchester, VT). When calculating the volume, the cross-sectional areas of region of interest (ROI) in each section was traced on the computer screen at low power using a $2 \times / 4 \times$ lens and the volume of ROI was calculated according to the Cavalieri principle (28). Sections encompass the whole striatum rostrally from the genu of the corpus callosum and caudally to the rostral part of the hippocampus corresponding to plates 9-24 of Paxinos' rat brain atlas (29). For the ROI, right and left hemisphere and SVZ were traced. Morphologic criteria were used consistently in all animals to determine the boundaries of SVZ. Dorsolateral striatal extension of SVZ, which resembles the dark band and thin long triangle in the sections corresponding to plates 10-20, was outlined. Briefly, the superior-medial boundary of SVZ was defined by the corpus callosum, the lateral boundary by the striatum, and the inferior boundary by the lateral ventricular margin. By using this sampling strategy, approximately 11 histologic sections per brain for hemispheric measurement and approximately 6 sections per brain for SVZ measurement were analyzed. Quantification was conducted blind by an examiner unaware of treatment group. Damage due to stroke was determined quantitatively by calculating the percentage preserved volume in the lesioned versus the control hemisphere. Size alteration of SVZ was investigated by calculating the percent SVZ volume in lesioned versus control hemisphere in each animal.

Data analysis. Data are presented as mean \pm SD. For statistical analysis, nonparametric methods were used. Kruskal-Wallis test was done for comparisons of multiple groups followed by the Wilcoxon rank sum test including a Bonferroni adjustment for comparison between two groups. Pearson correlation coefficients compared statistical relationships. All statistical analyses described above were done using SAS software program version of 6.12 (SAS Institute, Cary, NC). $p$ Values below 0.05 were considered significant.

\section{RESULTS}

Injury assessment. For this study, 16 animals received transient MCAO and sham surgery was performed in 8 animals. According to the inclusion criterion by imaging of diffusion weighted MRI, 13 of 16 animals were included and allocated randomly to MCAO with vehicle $(n=7)$ or MCAO with EPO treatment $(n=6)$. Most of the pups at P10 given MCAO showed poor sucking, especially for the first 2 or $3 \mathrm{~d}$ after surgery. One pup from the EPO-treated MCAO group and two pups from the vehicle-treated MCAO group died during the following $2 \mathrm{wk}$ before the analysis. No animal died during or immediately after the procedure. Therefore, 18 animals survived; sham surgery with vehicle $(n=4)$, sham surgery 
with EPO $(n=4)$, MCAO with vehicle $(n=5)$, and MCAO with EPO $(n=5)$.

Animals given transient MCAO showed significant tissue loss in the striatum and parietotemporal cortex ipsilateral to MCAO at 2 wk after surgery. For quantitative measurement of injury severity, the ratio of intact ipsilateral hemisphere volume to contralateral hemisphere volume was calculated. Despite reports that EPO promotes neurogenesis, in the animals given sham surgery, EPO treatment did not affect the volume of the hemispheres (Fig. 1A). In vehicle-treated animals at $2 \mathrm{wk}$ after MCAO surgery, there was significant decrease in percentage preserved volume compared with sham animals $(p<0.05)$. Animals that received EPO had a marked protective effect compared with vehicle treated MCAO animals $(p<0.05)$ (Fig. $1 B)$.

Assessment of SVZ changes. Cresyl Violet-stained sections revealed the definite demarcation of SVZ in the dorsolateral aspect of the lateral ventricle (Fig. 2). In sham animals treated with vehicle (Fig. 2, $A$ and $E$ ) versus those treated with EPO (Fig. 2, $B$ and $F$ ), there were no differences in the morphology of SVZ. In the rostral section (corresponding to Plate 10 of Paxinos' rat brain atlas) (29), the SVZ revealed a narrow, intensely stained band between the corpus callosum and subcallosal striatum adjacent to the striatal ventricular margin. The SVZ widened dorsolaterally and showed a dorsolateral tail beneath the corpus callosum through caudal sections (corresponding to Plate 20 of Paxinos' rat brain atlas) (29). Transient MCAO induced remarkable morphologic changes in the ipsilateral SVZ, with marked expansion rostrally and laterally in more rostral sections (Fig. 2C), and showed a more triangular shape in more caudal sections resulting from ipsilateral striatal atrophy and corresponding widening of the lateral ventricle (Fig. 2G). EPO treatment significantly decreased these morphologic changes (Fig. 2, $D$ and $H$ ). When we measured the SVZ volume on the contralateral side to the MCAO or in shams, there were no statistical differences among the four groups (data not shown). To evaluate the degree of expansion of ispsilateral SVZ, the percentage volume ratio of SVZ ipsilateral over contralateral to MCAO was calculated. Vehicle-

A

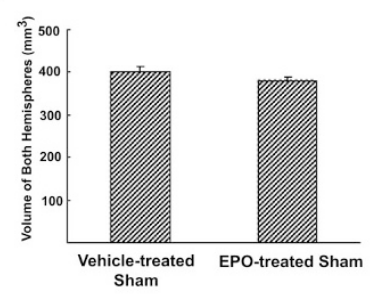

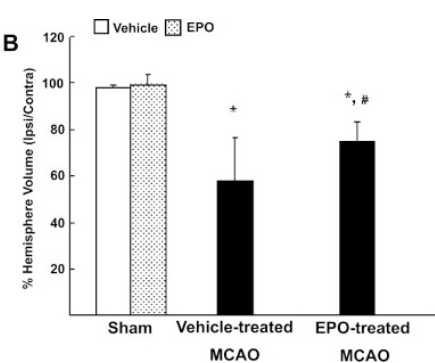

Figure 1. Stereologic volumetric quantification for brain damage. EPO did not affect brain volume in sham-operated animals $(A)$. Preserved volume in the ipsilateral hemisphere was expressed as a ratio of intact ipsilateral tissue volume over the contralateral hemisphere $(B)$. Transient MCAO in immature P10 rats caused significant reduction in percentage volume at 2 wk after surgery. (EPO significantly increased preserved volume after transient MCAO. $* p<0.05$, compared with sham animals, \#p<0.05, compared with vehicletreated MCAO animals). Data shown as mean $\pm \mathrm{SD} ; n=4$ for vehicle-treated sham, $n=4$ for EPO-treated sham, $n=5$ for vehicle-treated MCAO, $n=5$ for EPO-treated MCAO. treated MCAO animals showed significantly increased percentage volume of ispilateral SVZ compared with the sham operated animals $(p<0.05)$. EPO treatment significantly decreased this ipsilateral SVZ enlargement $(p<0.05)$ (Fig. $3 A$ ). For the evaluation of the relationship between the degree of SVZ expansion and the degree of tissue damage, the ratio of percentage hemispheric volume and percentage SVZ volume as described was compared and revealed an inverted linear relationship with the coefficients of simple determination of $0.3525\left(r^{2}\right)(p<0.02)$ (Fig. 3B).

Behavior test with cylinder rearing. To evaluate the lateralizing sensorimotor deficit, the percentage of weight-bearing episodes that were initiated with the nonimpaired (ipsilateral) forepaw (Fig. 4A) and the percentage difference between nonimpaired (ipsilateral) and impaired (contralateral) forepaw initiation (Fig. 4B) was calculated in each animal at P24 before sacrificing them for brain evaluation. As expected, sham animals showed symmetrical use of bilateral paw when rearing, and percentage ipsilateral and contralateral paw initiation was around $20 \%$.

MCAO animals showed marked asymmetry due to the preference for the use of nonimpaired, ipsilateral paw when rearing, compared with sham $(p<0.01)$. EPO treatment significantly improved this asymmetry compared with vehicle-treated MCAO animals $(p<0.05)$. When we compared the percentage hemisphere volume ipsilateral versus contralateral and percent-

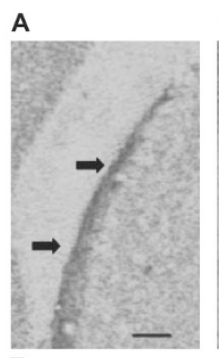

E

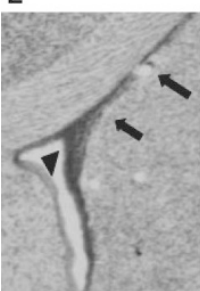

B

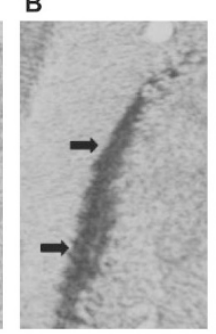

$\mathbf{F}$

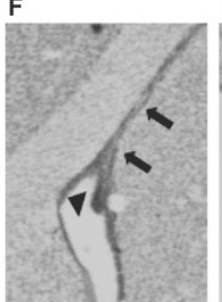

C

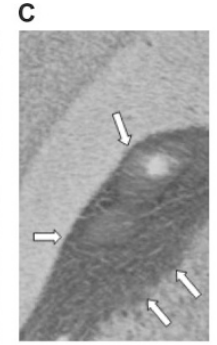

G

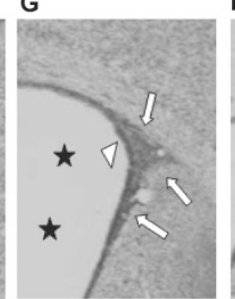

D

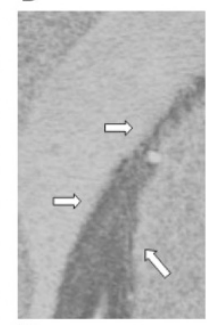

H

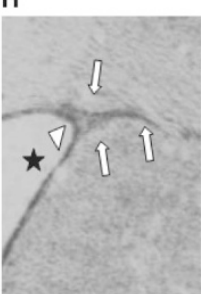

Figure 2. SVZ morphology in cresyl-violet stained sections of the ipsilateral brain of rats at $2 \mathrm{wk}(\mathrm{P} 24)$ after surgery. Upper row represents sections including rostral part of striatal SVZ corresponding to Plate 11 of Paxinos' rat brain atlas. Lower row represents sections including mid-striatal SVZ corresponding to plates 16 or 17 of Paxinos' rat brain atlas. In vehicle- $(A, E)$ or EPO- $(B, F)$ treated sham animals, $\mathrm{SVZ}$ is recognized as a densely stained dark band-like structure residing between the corpus callosum and subcallosal striatum in rostral part sections (black arrows). The SVZ widens and appears triangular with the inferior boundary being the lateral ventricular margin (black arrowhead). Transient MCAO induced changes in ipsilateral SVZ. In vehicletreated MCAO animals $(C, G), \mathrm{SVZ}$ in ischemic hemisphere expands markedly, especially in the rostral sections ( $C$, white arrows). Mid-striatal SVZ $(G)$ shows a more triangular shape with broader inferior base (white arrowhead) along the lateral ventricular margin due to the enlargement of the ventricle (black stars) because of severe striatal atrophy. EPO treatment significantly decreased these morphologic changes of SVZ after $\operatorname{MCAO}(D, H)$. Scale bar $=100 \mu \mathrm{m}$. 


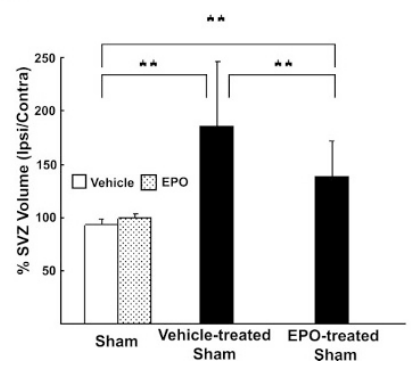

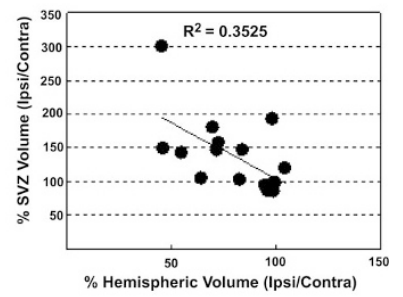

Figure 3. Stereologic volumetric changes in SVZ in the ischemic hemisphere expressed as a percentage of the contralateral hemisphere. EPO did not affect the SVZ volume in sham-operated animals (data not shown). Vehicle-treated MCAO animals showed markedly increased expansion of SVZ with respect to sham-operated animals. EPO treatment significantly ameliorates these changes after MCAO $(A)\left(^{* *} p<0.05\right.$ in Wilcoxon rank sum test between each two group. Data shown as mean $\pm \mathrm{SD} ; n=4$ for vehicle-treated sham, $n=4$ for EPO-treated sham, $n=5$ for vehicle-treated MCAO, $n=5$ for EPO-treated MCAO). Regression analysis $(B)$ showed an inverse relationship between percentage preserved volume and ipsilateral expansion of SVZ $\left(r^{2}=0.3525\right.$, $p<0.02)$.

age nonimpaired limb contacts in the cylinder test, a direct inverted linear relationship was seen with the coefficients of simple determination $\left(r^{2}\right)$ of $0.8121(p=0.0001)$ (Fig. 4C).

\section{DISCUSSION}

These results show that EPO is protective after ischemic stroke in immature animals, both by ameliorating brain tissue loss and improving sensorimotor function. This study also indicates that focal ischemia-reperfusion injury increases the size of the ipsilateral forebrain neonatal SVZ, suggesting that precursor proliferation is involved in the response of the neonatal brain to ischemic injury. Furthermore, this effect is directly correlated with injury severity, such that animals with more severe injury had a greatly expanded SVZ. Therefore, attenuated expansion of forebrain SVZ in EPO-treated animals in this study can be explained by injury reduction exerted by EPO after transient MCAO in immature rats. Importantly, systemic EPO did not have a growth factor effect in normal or sham operated brains.

EPO is known as one of the major target genes of hypoxia inducible factor 1 (HIF-1), an oxygen-sensing molecule in tissue including brain $(30,31)$. We previously demonstrated that HIF-1 is induced in our model (4). Expression of EPO and EPO receptor has been demonstrated in ischemic neonatal brain after hypoxia-ischemia (12) and permanent MCAO (13). The mechanism of EPO protection in ischemic brain in the mature animal is thought to be due to penumbral activation of EPO receptor. Binding of EPO to its receptor generates an anti-apoptotic cascade $(9,32,33)$. Therefore, if the penumbral region is larger, the cytoprotectant effect of EPO will be greater (34). In the adult rodent, whereas EPO significantly decreased the infarct size after transient MCAO (8), histologic reduction of infarct size was not seen after more severe injury as in permanent MCAO (17).

As our group reported previously, in this transient stroke model in an immature animal, there is no clear demarcation of penumbra and core in injured tissue (35). Neuronal caspase-3

activation occurred robustly in both the ischemic core and penumbra in our P7 rat model. These findings support the speculation that the cytoprotection by EPO through antiapoptotic action may be greater in immature brain after transient stroke. The mechanism of EPO protection must be clarified with further study.

Several studies have reported that systemic administration of rhEPO reduced brain injury in neonatal models, including hypoxia-ischemia $(7,10-12,15)$ and $N$-methyl-D-aspartate receptor antagonist neurotoxicity (36). Although these studies have reported tissue protection with EPO treatment, a sensorimotor functional outcome has not been evaluated. Forepaw placement in cylinder rearing is a well-known test in the adult rodent as a measure of forelimb function (37-40). Particularly, asymmetry of forelimb wall contact is considered an index of motor system integrity and is widely used in adult rodent MCAO models $(39,40)$. The cylinder test is simple, reliable, and reproducible. However, possible limitations have to be considered in the detection of lateralizing sensorimotor deficits originating in the neonatal period. At this age, animals are still immature in regard to motor function. Another limitation is that, with the serial monitoring for function over time, the sensitivity of the test can be decreased due to learning and functional recovery during that period (41). Grow et al. (26) have reported that the cylinder test in P35 rat could detect sensorimotor deficits after hypoxia-ischemia and correlated well with cortical striatal damage. We performed the cylinder test at P24 and revealed that it was sensitive enough to detect limb use asymmetry at this age and the asymmetry of limb use directly correlated with the extent of brain damage. To our knowledge, this study is the first report of improvement in sensorimotor function by EPO treatment in neonatal animals after ischemic brain damage. For the documentation of longterm functional recovery, follow-up studies are underway.

The SVZ in rodent forebrain contains neuronal precursor cells throughout life. Various types of injury stimulate the proliferation of SVZ precursor cells in adult rodent brain, and it is believed that stem cells in neurogenic areas respond to injury (42). Increase in size of SVZ and proliferation of neuronal precursor cells has been demonstrated in adult rodent after MCAO $(19,20)$. Several studies suggest that specific growth or neurotrophic factors, such as IGF-1 and brainderived neurotrophic factor (BDNF), influence neural precursor proliferation in the adult rodent brain $(43,44)$. Inflammatory reactions in the CNS can produce various neurotrophic factors such as brain-derived neurotrophic factor, nerve growth factor, and neurotrophins 3 and 4/5 (45). These observations support our results demonstrating that focal ischemia-reperfusion injury stimulates SVZ expansion in neonatal rodent brain. Our finding also corresponds with the recent report of Plane et al. (23) that showed a significant increase in SVZ area after hypoxia-ischemia in P10 mice.

$\mathrm{EPO}$ is also known to have neurotrophic actions both in vivo and in vitro (16). For example, EPO treatment increases proliferation of precursor cells of SVZ area in permanent MCAO in adult rodent (17). However, in that study, EPO did not decrease the infarct size, which is an important difference from our study. We demonstrated that the animals with more injury 
A

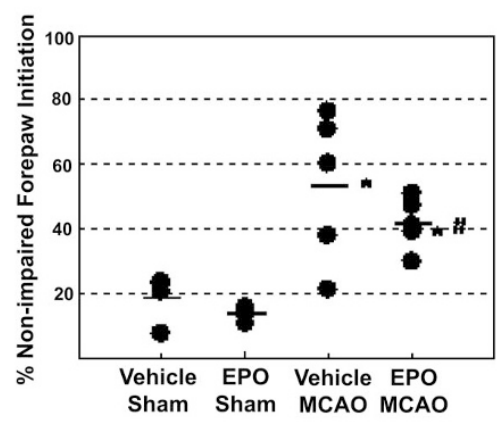

B

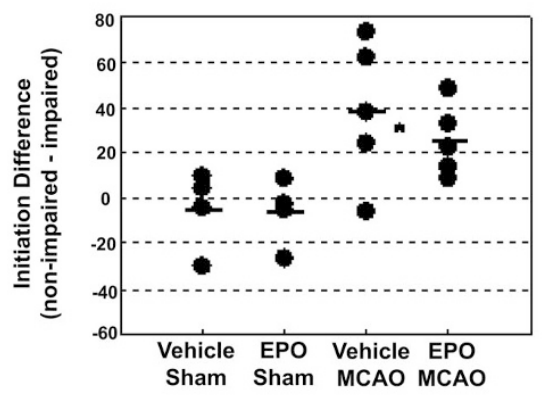

C

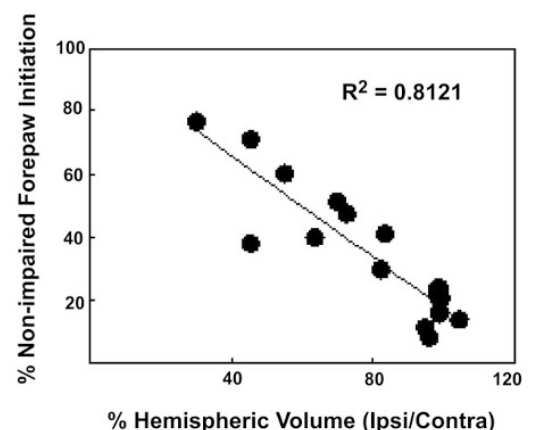

Figure 4. Behavioral testing at 2 wk (P24) after surgery in P10 rats. Percentage weight-bearing contacts initiated with the nonimpaired (ipsilateral) forepaw in cylinder rearing $(A)$ and percentage difference between nonimpaired (ipsilateral) and impaired (contralateral) forepaw initiation $(B)$ of each animal were plotted in the four different groups. Vehicle-treated MCAO animals showed marked forelimb use asymmetry by revealing preferential use for nonimpared, ipsilateral limb. EPO treatment significantly decreased this functional asymmetry after transient MCAO. Data are shown as individual values from each animal; bar represents the mean for each group [ ${ }^{*} p<0.01$ in $(A), p<0.05$ in $(B)$, compared with vehicle-treated sham animals; $\# p<0.05$, compared with vehicle-treated MCAO animals; $n=4$ for vehicle sham, $n=4$ for EPO sham, $n=5$ for vehicle MCAO, $n=5$ for EPO MCAO). Regression analysis (C) of percentage nonimpaired limb initiation in the cylinder test and percentage preserved hemispheric volume shows that forelimb use asymmetry is directly correlated with degree of brain damage $\left(r^{2}=0.8121, p=0.0001\right)$.

after transient stroke had a greatly increased SVZ. After hypoxia-ischemia, increases in SVZ area were directly correlated with the extent of tissue injury (23).

Levison et al. (21) reported that hypoxia-ischemia depleted the SVZ and precursor cells. The differences from our results may be derived from the injury severity and model difference. Another difference may be derived from the method used to evaluate SVZ. We evaluated the volume of SVZ including the entire rostral to caudal extent, whereas the study of Levison $e t$ al. evaluated the stem cell population of SVZ mainly at the level of the anterior commissure, which is the more caudal part of SVZ. Recently Romanko et al. (47) reported that the medial part of dorsolateral SVZ is resistant to hypoxic-ischemic injury. In this study, we calculated the total SVZ volume using stereologic principles that are unaffected by the possibility of uneven sectioning through damaged tissue and compared ipsilateral versus contralateral to correct for interanimal differences in SVZ size. We observed that the rostral part of SVZ is more markedly expanded compared with the caudal part of SVZ, because we cannot detect size difference caudally because of marked changes in morphology due to enlargement of the lateral ventricle. Caution is needed in interpreting the effect of EPO on neonatal SVZ after ischemic injury, because we investigated only the morphologic change of SVZ, not the proliferation of precursor cells in SVZ.

In our study, two hypotheses may be considered for these actions of EPO. First, EPO is directly cytoprotective and preserved brain tissue is responsible for improved functional outcome. Second, EPO stimulates neurogenesis, repopulating injured brain tissue and improving functional outcome. Our observation that EPO attenuated expansion of forebrain SVZ is more consistent with the first hypothesis. However we cannot rule out the possibility that EPO influences cell fate decisions among the progeny of mitotic precursor cells in the SVZ, shifting the response toward the generation of neurons. Studies to answer this important question are underway. Of course, these two hypotheses are not mutually exclusive and both may represent important mechanisms for protection and recovery after neonatal ischemic brain injury.

We did not examine the effect of EPO on erythropoiesis in this study. A recent report has shown that derivatives of EPO can be tissue protective but not erythropoietic, which may be detrimental following stroke (46).

In our model of neonatal ischemic stroke, proven by diffusion weighted MRI, EPO preserved brain volume and improved sensorimotor function. EPO therefore is an excellent candidate for the treatment of neonatal stroke, one of the most devastating conditions occurring during the neonatal period.

\section{REFERENCES}

1. Lynch JK, Nelson KB 2001 Epidemiology of perinatal stroke. Curr Opin Pediatr 13:499-505

2. Nelson KB, Lynch JK 2004 Stroke in newborn infants. Lancet Neurol 3:150-158

3. Derugin N, Wendland M, Muramatsu K, Roberts TP, Gregory G, Ferriero DM, Vexler ZS 2000 Evolution of brain injury after transient middle cerebral artery occlusion in neonatal rats. Stroke 31:1752-1761

4. Mu D, Jiang X, Sheldon RA, Fox CK, Hamrick SE, Vexler ZS, Ferriero DM 2003 Regulation of hypoxia-inducible factor 1alpha and induction of vascular endothelial growth factor in a rat neonatal stroke model. Neurobiol Dis 14:524-534

5. Sadamoto Y, Igase K, Sakanaka M, Sato K, Otsuka H, Sakaki S, Masuda S, Sasaki R 1998 Erythropoietin prevents place navigation disability and cortical infarction in rats with permanent occlusion of the middle cerebral artery. Biochem Biophys Res Commun 253:26-32

6. Bernaudin M, Marti HH, Roussel S, Divoux D, Nouvelot A, MacKenzie ET, Petit E 1999 A potential role for erythropoietin in focal permanent cerebral ischemia in mice. J Cereb Blood Flow Metab 19:643-651

7. Aydin A, Genc K, Akhisaroglu M, Yorukoglu K, Gokmen N, Gonullu E 2003 Erythropoietin exerts neuroprotective effect in neonatal rat model of hypoxicischemic brain injury. Brain Dev 25:494-498

8. Brines ML, Ghezzi P, Keenan S, Agnello D, de Lanerolle NC, Cerami C, Itri LM, Cerami A 2000 Erythropoietin crosses the blood-brain barrier to protect against experimental brain injury. Proc Natl Acad Sci U S A 97:10526-10531

9. Siren AL, Fratelli M, Brines M, Goemans C, Casagrande S, Lewczuk P, Keenan S, Gleiter C, Pasquali C, Capobianco A, Mennini T, Heumann R, Cerami A, Ehrenreich H, Ghezzi P 2001 Erythropoietin prevents neuronal apoptosis after cerebral ischemia and metabolic stress. Proc Natl Acad Sci U S A 98:4044-4049

10. Matsushita H, Johnston MV, Lange MS, Wilson MA 2003 Protective effect of erythropoietin in neonatal hypoxic ischemia in mice. Neuroreport 14:1757-1761

11. Kumral A, Ozer E, Yilmaz O, Akhisaroglu M, Gokmen N, Duman N, Ulukus C, Genc S, Ozkan H 2003 Neuroprotective effect of erythropoietin on hypoxic-ischemic brain injury in neonatal rats. Biol Neonate 83:224-228

12. Sun Y, Zhou C, Polk P, Nanda A, Zhang JH 2004 Mechanisms of Erythropoietininduced Brain Protection in Neonatal Hypoxia-Ischemia Rat Model. J Cereb Blood Flow Metab 24:259-270 
13. Wen TC, Rogido M, Genetta T, Sola A 2004 Permanent focal cerebral ischemia activates erythropoietin receptor in the neonatal rat brain. Neurosci Lett 355:165-168

14. Sola A, Rogido M, Genetta T, Wen TC 2004 Erythropoietin prevents brain injury in neonatal rats with focal cerebral ischemia. Pediatr Res 55:434A

15. Kumral A, Uysal N, Tugyan K, Sonmez A, Yilmaz O, Gokmen N, Kiray M, Genc S, Duman N, Koroglu TF, Ozkan H, Genc K 2004 Erythropoietin improves long-term spatial memory deficits and brain injury following neonatal hypoxia-ischemia in rats. Behav Brain Res 153:77-86

16. Shingo T, Sorokan ST, Shimazaki T, Weiss S 2001 Erythropoietin regulates the in vitro and in vivo production of neuronal progenitors by mammalian forebrain neural stem cells. J Neurosci 21:9733-9743

17. Wang L, Zhang Z, Wang Y, Zhang R, Chopp M 2004 Treatment of stroke with erythropoietin enhances neurogenesis and angiogenesis and improves neurological function in rats. Stroke 35:1732-1737

18. Lois C, Garcia-Verdugo JM, Alvarez-Buylla A 1996 Chain migration of neuronal precursors. Science 271:978-981

19. Arvidsson A, Collin T, Kirik D, Kokaia Z, Lindvall O 2002 Neuronal replacemen from endogenous precursors in the adult brain after stroke. Nat Med 8:963-970

20. Parent JM, Vexler ZS, Gong C, Derugin N, Ferriero DM 2002 Rat forebrain neurogenesis and striatal neuron replacement after focal stroke. Ann Neurol 52:802813

21. Levison SW, Rothstein RP, Romanko MJ, Snyder MJ, Meyers RL, Vannucci SJ 2001 Hypoxia/ischemia depletes the rat perinatal subventricular zone of oligodendrocyte progenitors and neural stem cells. Dev Neurosci 23:234-247

22. Skoff RP, Bessert DA, Barks JD, Song D, Cerghet M, Silverstein FS 2001 Hypoxicischemic injury results in acute disruption of myelin gene expression and death of oligodendroglial precursors in neonatal mice. Int J Dev Neurosci 19:197-208

23. Plane JM, Liu R, Wang TW, Silverstein FS, Parent JM 2004 Neonatal hypoxicischemic injury increases forebrain subventricular zone neurogenesis in the mouse. Neurobiol Dis 16:585-595

24. Hagberg H, Bona E, Gilland E, Puka-Sundvall M 1997 Hypoxia-ischaemia model in the 7-day-old rat: possibilities and shortcomings. Acta Paediatr Suppl 422:85-88

25. Derugin N, Ferriero DM, Vexler ZS 1998 Neonatal reversible focal cerebral ischemia: a new model. Neurosci Res 32:349-353

26. Grow JL, Liu YQ, Barks JD 2003 Can lateralizing sensorimotor deficits be identified after neonatal cerebral hypoxia-ischemia in rats? Dev Neurosci 25:394-402

27. Schallert T, Fleming SM, Leasure JL, Tillerson JL, Bland ST 2000 CNS plasticity and assessment of forelimb sensorimotor outcome in unilateral rat models of stroke, cortical ablation, parkinsonism and spinal cord injury. Neuropharmacology 39:777-787

28. Regeur L, Pakkenberg B 1989 Optimizing sampling designs for volume measurements of components of human brain using a stereological method. J Microsc 155:113-121

29. Paxinos G 1986 The Rat Brain in Stereotaxic Coordinates. Academic Press, San Diego, CA

30. Bracken CP, Whitelaw ML, Peet DJ 2003 The hypoxia-inducible factors: key transcriptional regulators of hypoxic responses. Cell Mol Life Sci 60:1376-1393

31. Sharp FR, Bernaudin M 2004 HIF1 and oxygen sensing in the brain. Nat Rev Neurosci 5:437-448

32. Digicaylioglu M, Lipton SA 2001 Erythropoietin-mediated neuroprotection involves cross-talk between Jak2 and NF-kappaB signalling cascades. Nature 412:641-647
33. Chong ZZ, Kang JQ, Maiese K 2002 Erythropoietin is a novel vascular protectant through activation of Akt1 and mitochondrial modulation of cysteine proteases. Circulation 106:2973-2979

34. Grasso G, Sfacteria A, Cerami A, Brines M 2004 Erythropoietin as a tissue-protective cytokine in brain injury: what do we know and where do we go? Neuroscientist 10:93-98

35. Manabat C, Han BH, Wendland M, Derugin N, Fox CK, Choi J, Holtzman DM, Ferriero DM, Vexler ZS 2003 Reperfusion differentially induces caspase-3 activation in ischemic core and penumbra after stroke in immature brain. Stroke 34:207-213

36. Dzietko M, Felderhoff-Mueser U, Sifringer M, Krutz B, Bittigau P, Thor F, Heumann R, Buhrer C, Ikonomidou C, Hansen HH 2004 Erythropoietin protects the developing brain against $\mathrm{N}$-methyl-D-aspartate receptor antagonist neurotoxicity. Neurobiol Dis 15:177-187

37. Murray M, Kim D, Liu Y, Tobias C, Tessler A, Fischer I 2002 Transplantation of genetically modified cells contributes to repair and recovery from spinal injury. Brain Res Brain Res Rev 40:292-300

38. DeBow SB, Davies MLA, Clarke HL, Colbourne F 2003 Constraint-induced movement therapy and rehabilitation exercises lessen motor deficits and volume of brain injury after striatal hemorrhagic stroke in rats. Stroke 34:1021-1026

39. Karhunen H, Virtanen T, Schallert T, Sivenius J, Jolkkonen J 2003 Forelimb use after focal cerebral ischemia in rats treated with an alpha 2-adrenoceptor antagonist Pharmacol Biochem Behav 74:663-669

40. Biernaskie J, Chernenko G, Corbett D 2004 Efficacy of rehabilitative experience declines with time after focal ischemic brain injury. J. Neurosci 24:1245-1254

41. Felt BT, Schallert T, Shao J, Liu Y, Li X, Barks JD 2002 Early appearance of functional deficits after neonatal excitotoxic and hypoxic-ischemic injury: fragile recovery after development and role of the NMDA receptor. Dev Neurosci 24:418425

42. Lie DC, Song H, Colamarino SA, Ming GL, Gage FH 2004 Neurogenesis in the adult brain: new strategies for central nervous system diseases. Annu Rev Pharmacol Toxicol 44:399-421

43. Aberg MA, Aberg ND, Hedbacker H, Oscarsson J, Eriksson PS 2000 Peripheral infusion of IGF-I selectively induces neurogenesis in the adult rat hippocampus. J Neurosci 20:2896-2903

44. Pencea V, Bingaman KD, Wiegand SJ, Luskin MB 2001 Infusion of brain-derived neurotrophic factor into the lateral ventricle of the adult rat leads to new neurons in the parenchyma of the striatum, septum, thalamus, and hypothalamus. J Neurosci 21:6706-6717

45. Kerschensteiner M, Stadelmann C, Dechant G, Wekerle H, Hohlfeld R 2003 Neurotrophic cross-talk between the nervous and immune systems: implications for neurological diseases. Ann Neurol 53:292-304

46. Leist M, Ghezzi P, Grasso G, Bianchi R, Villa P, Fratelli M, Savino C, Bianchi M, Nielsen J, Gerwien J, Kallunki P, Larsen AK, Helboe L, Christensen S, Pedersen LO, Nielsen M, Torup L, Sager T, Sfacteria A, Erbayraktar S, Erbayraktar Z, Gokmen N, Yilmaz O, Cerami-Hand C, Xie QW, Coleman T, Cerami A, Brines M 2004 Derivatives of erythropoietin that are tissue protective but not erythropoietic. Science 305:239-242

47. Romanko MJ, Rothstein RP, Zevison SW 2004 Neural stem cells in the subventricular zone are resilient to hypoxia/ischemia whereas for progenitors are vulnerable. J Cereb Blood Flow Metab 24:814-825 